sample values. The patient had reccurent problems with social functioning, which manifested as intermittent palpitations, precordial sensations, and a prolonged episode of large amplitude incontrollable tremor for which he was hospitalized once again after the second episode of pancreatitis.

Conclusion The presented cases show the possible extent of extrapulmonary illness caused by Mycoplasma pneumoniae in children. Judging by these cases, autoimmune mechanisms and also genetic factors seem to play an important role in the infection of a specific organ by $\mathrm{M}$. pneumoniae.

\section{REFEEDING SYNDROME IN HOSPITALIZED CHILDREN AND ADOLESCENTS WITH EATING DISORDERS- EXPERIENCE OF TERTIARY CENTER}

Barbara Perše*, Ana Kovačević, Andrijana Kološa, Matea Crnković Ćuk, Bruno Špiljak, Luka Šimunović, Orjena Žaja. Sestre Milosrdnice University Hospital Center (Zagreb, Croatia)

10.1136/archdischild-2021-europaediatrics. 255

Refeeding syndrome (RFS) describes potentially fatal shift in electrolytes in severe malnourished patients receiving rapid and excessive food re-introduction. It is a result of hormonal and metabolic disturbances.

There are various clinical and laboratory features with hypophosphatemia being the most common one. Elevation in liver function tests is also frequently seen. Patients with anorexia are a high-risk group for developing RFS. The aim of the study was to investigate the incidence and clinical features of RFS among hospitalized patients, as well as severity of malnutrition (Z-score, BMI).

This study is a retrospective analysis of medical documentation of patients diagnosed with anorexia nervosa (restricting (ANRT) and binge eating/purging

(ANBP) subtype), eating disorder NOS (EDNOS) and avoidant/restrictive food intake disorder (ARFID) who were admitted to our Centre for eating disorders in children and adolescents during a 5 year period (2014-2018). We analyzed the age, gender, duration of the disease before admittance, anthropometric data (BMI and Z-score), average weight loss, the need for nasogastric (NG) tube feeding and phosphate supplementation. For statistical analysis we used t-test.

256 patients (232 female) aged 6-20 years (median 15+/2.06 ) of which $43 \%$ were diagnosed with ANRT, 10\% ANBP, 8\% ARFID, 39\% with EDNOS were included in the study. The average duration of the disease at the time of admittance was 13.25+/-13.43 months. Average BMI Z-score was -1.97 $+/-1.63$ average weight loss was $20+/-9.76 \%$ initial body weight (IBW).

Hypophosphatemia was found in $15.6 \%$ patients, of which $65 \%$ received phosphate supplements by oral or intravenous route depending of phosphate serum concentration. Elevated liver enzymes due to RFS were found in $9.3 \%$ of patients. In total RFS in some form developed in 23\% patients.

Average BMI Z-score of patients that developed RFS was $2.6+/-1.89$, average weight loss was $23.9+/-9.85 \%$ of IBW. Both variables were significantly different $(p<0.05)$ in comparison with non-RFS group which had BMI Z-score of

$-1.76+/-1.47$ and average weight loss of $18.7+/-9.3 \%$ of IBW. The average duration of the disease was similar in both groups (13 months) $(\mathrm{p}=0.84)$.
NG tube feeding was needed in $27 \%$ of all patients, $24 \%$ patients in non-RFS group and $37 \%$ in patients with RFS.

Our study reported that even in controlled hospital conditions and with careful realimentation RFS has a high incidence. We found statistically significant difference when it comes to BMI Z-score and average weight loss between two groups of patients.

\section{HEMOPHAGOCYTIC LYMPHOHISTIOCYTOSIS IN A 13- YEAR OLD GIRL WITH CROHN'S DISEASE}

Barbara Perše*, Marina Mataija, Matej Katavić, Ika Kardum-Skelin, Matea Crnković Ćuk, Orjena Žaja. Sestre Milosrdnice University Hospital Center

\subsection{6/archdischild-2021-europaediatrics.256}

Hemophagocytic lymphohistiocytosis (HLH) is a severe, lifethreatening disorder of uncontrolled immune activation and inflammation which, if left untreated, can lead to organ failure and death. Mortality is high, so prompt recognition and treatment are crucial. However, clinical presentation is unspecific, condition is rare, so in some cases the diagnosis and treatment can be delayed, which further impairs chances of recovery. In recent years, the number of reported cases of HLH in inflammatory bowel disease (IBD) patients has increased, probably due to higher awareness than real rise in incidence, although this condition is still often overlooked.

We describe a 13-year-old girl with Crohn's disease (CD) on azathioprine therapy, who developed HLH as a complication of primary CMV infection and EBV reactivation.

13-year-old girl was diagnosed with $\mathrm{CD}$ two years prior and was in stable remission with standard pediatric therapy, enteral therapy, followed by azathioprine maintenance therapy, which she was currently taking. The function of TPMT was normal.

Two months after last follow up she was admitted in poor clinical condition with a prolonged period of high-grade fever, fatigue, poor appetite, weight loss, few episodes of vomiting associated with peaks of fever and slight abdominal tenderness. No other symptoms suggestive of CD relapse were present which was later confirmed by normal ileocolonoscopy and MR enterography. According to history she was in contact with infectious mononucleosis patient two months prior. Azathioprine was discontinued. After obtaining specimens for microbiological analysis empirical broad-spectrum antibiotics were initiated. During next few days her clinical state started to deteriorate. At this point, additional tests confirmed our suspicion of

HLH: persistent fever, bicytopenia, hyperferritinemia, hypofibrinogenemia, hypertriglyceridemia, low NK activity, splenomegaly, and CD68 and ANA positive phagocytes in bone marrow smear. All bacterial cultures came back negative. Serology and PCR results revealed that HLH was a complication of primary CMV infection with EBV reactivation.

Treatment with systemic corticosteroids was initiated with excellent response, lithic drop of fever, improvement in general condition and normalization of biochemical markers. The dose of corticosteroids was tapered gradually and her clinical state was monitored closely. She had no signs of relapses of HLH, primary disease, nor no signs of malignant disease during one year of follow-up period. 